\title{
Multi-Market, Multi-Product New Product Diffusion: Decomposing Local, Foreign, and Indirect (Cross-Product) Effects
}

\author{
Antonio Ladrón-de-Guevara • William P. Putsis
}

Published online: 17 December 2014

(C) Springer Science+Business Media New York 2014

\begin{abstract}
In this study, we address an important issue largely ignored in existing diffusion research - the simultaneous diffusion of related (here, complementary) products across multiple interacting countries. In doing so, we demonstrate that incorporating prior diffusion of complementary products in an international framework leads to an enhanced substantive understanding of the evolution of cross-country diffusion. The limited prior research on cross-product interactions has focused exclusively on a single country. We extend this research by building a more complete view of the role that prior diffusion of two interacting technologies play both within as well as across countries. Specifically, we decompose - on a country-by-country basis - the impact of three factors on the diffusion of any product: (a) prior diffusion of the product within each country, (b) prior diffusion of the same product in other counties, and (c) prior diffusion of a related product. This decomposition leads to a number of important strategic insights. We estimate and graph the three effects over time for each product and country using a comprehensive data set that covers the diffusion of PCs and the Internet over two decades-from 1981 to 2009-and across 19 countries. There are a number of interesting findings. First, we find that home PC diffusion was driven predominantly by local effects - the more individuals saw the penetration of
\end{abstract}

Electronic supplementary material The online version of this article (doi:10.1007/s40547-014-0032-x) contains supplementary material, which is available to authorized users.

\footnotetext{
A. Ladrón-de-Guevara

Universitat Pompeu Fabra, C/Ramon Trias Fargas 25-27,

08004 Barcelona, Spain

e-mail: antonio.ladron@upf.edu

W. P. Putsis $(\bowtie)$

University of North Carolina, CB 3490, McColl Building, Chapel

Hill, NC 27599-3490, USA

e-mail: William_Putsis@unc.edu
}

home PCs grow locally, the greater the likelihood of adoption. Second, we find very different effects for the Internet-Internet adoption was driven by a combination of influences: (a) local network effects, (b) foreign network effects, and (c) cross-product effects. These results suggest that diffusion of one product can facilitate the diffusion of another product and that the impact can be asymmetric across products. When taken in aggregate, these results highlight the importance of incorporating and estimating cross-product effects in a multi-market new product diffusion context —one is able to obtain a more complete view of the impact of strategic decisions within a general diffusion process in markets that develop and evolve dynamically over time.

Keywords Multi-product diffusion · Complementary products $\cdot$ Network externalities $\cdot$ International diffusion

\section{Introduction}

In the interconnected world of today, fewer and fewer products are truly "stand alone." Smartphones require Internet connections; social media sites require a computer, smartphone, or tablet; adoption decisions for apps on Apple's App Store depend upon prior adoption of iPhones and/or iPads, for example. Even home heating and cooling systems, refrigerators, and televisions are enhanced by information connectivity and access. Thus, for many products today, the adoption decision for one product often depends crucially upon prior adoption of another. When the utility that consumers derive from adopting an innovation grows with the number of existing users, the technology is said to present network effects. Further complicating the matter, for some of these products, adoption decisions are dependent upon domestic or local networks, while for other products, it may be the size of the international global network that is most 
important—social interconnectivity (e.g., Facebook, WeChat) tends to be more "local" (defined here as within-country) in nature, whereas access to information (e.g., via the Internet) tends to be more global (across countries) in nature.

Research on the diffusion of innovations has resulted in a plethora of research (see, e.g., [24]) over the years - one area that has seen a fair amount of attention more recently has been the subject of cross-country (i.e., "Multi-Market") and spillover effects. For example, building on work by [23, 27] examined the pattern of interaction across countries. Dekimpe et al. [7-9] and Talukdar et al. [38] addressed global diffusion influences across countries. Stremersch and Tellis [35] examined differences in the pattern of growth across countries, while Tellis et al. [39] focused on the time to takeoff for waterfall versus sprinkler strategies, the relative role of economics versus culture, etc. In related research, Van Everdingen et al. [42] build on this by examining international spillover effects, estimating how one country's time to takeoff influences another's time to takeoff.

While we are beginning to form a more complete view of how prior diffusion and/or time to takeoff in one country influences the diffusion process in another country as well as what factors play a prime or ancillary role in the process and how strategic decisions can facilitate or retard diffusion in a multinational setting, this stream of research has focused exclusively on single, stand-alone products such as microwave ovens, clothes dryers, washing machines, and the like. For most companies, this offers little practical guidance strategically - for many products, the utility of adopting one product depends upon complementary products in the market (e.g., software available for hardware).

While prior empirical diffusion research has included products for which related products clearly played a role in the diffusion path, no existing research on cross-country diffusion incorporates interactions across products into the analysis. Accordingly, we build on prior research addressing the implications of cross-country effects by incorporating cross-product effects as well as network effects (both within-country and cross-country) into a general model and empirical analysis. We argue that in order to fully understand the diffusion of products in an international setting, it is often essential to consider direct (same product) as well as cross-product interactions - in addition to cross-country relationships. Doing so within a single modeling framework that nests more traditional models enables us to discern additional insights, insights that could not be gained using single-product models alone.

As such, we believe that our approach makes a number of important contributions to the literature on diffusion and new product strategy in a multinational setting that are descriptive and conceptual in nature. First, by considering the impact of direct as well as cross (complementary) product effects both within and across countries, we build on recent work on crosscountry diffusion (e.g., [27,42]), addressing a call for such research made in a review by Dekimpe et al. [9]. We show that incorporating cross-product effects lead to an enhanced understanding of the evolution of cross-country diffusion. Second, previous empirical research on multi-product interactions in the case of complementary goods in a global context does not exist. The small amount of previous research has used survey data (e.g., [13]) or focused on cross-product effects within a single country $[4,6,16,36]$. Our research complements previous work by building a more complete view of the role that the adoption levels of two interacting technologies play both within as well as across countries, an understanding that has important strategic implications. For example, we suggest that foreign network effects and complementary product effects can offer an additional explanation for the "threshold" takeoff explanation offered by [13], thereby suggesting that strategy promoting foreign effects in order to facilitate threshold attainment can be particularly effective. Overall, we argue that by developing a comprehensive model that allows for diffusion processes (with covariates such as price) across countries that incorporates two interacting technologies, we are able to form a more complete picture beyond what previous research on cross-country diffusion and the literature on multi-product network effects has been able to provide. Thus, the main objective of this paper is descriptive in nature: to document and understand this process across countries and time, using the market for PCs and the Internet as an empirical example.

Noting that the framework can be applied in a variety of settings, we estimate our model using data on PC and Internet penetration as an empirical illustration/application. The data cover over two decades (1981-2009) and 19 countries in North America and Europe. Figure 1 depicts the aggregate

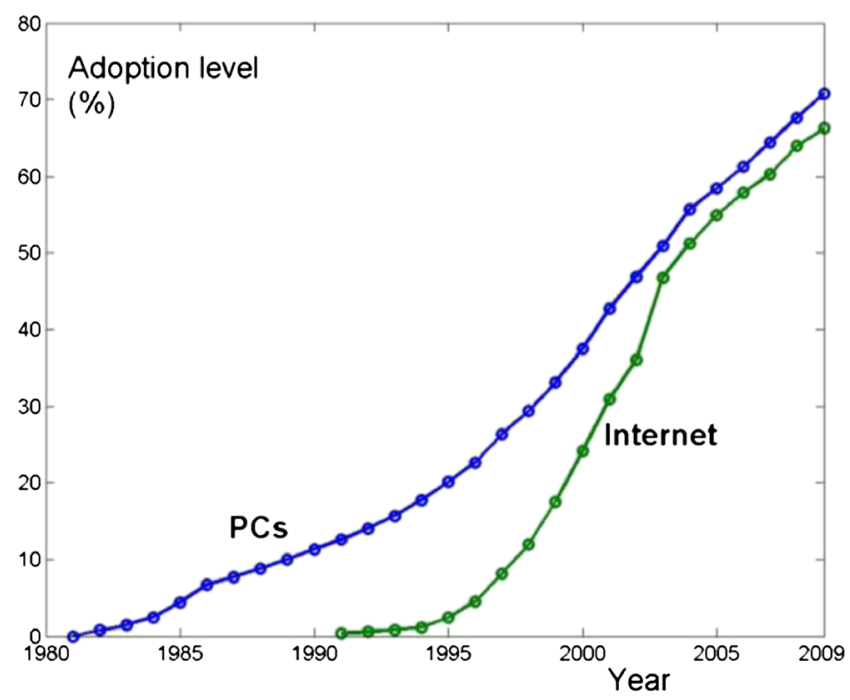

Fig. 1 Adoption levels for home PCs and the Internet (aggregate diffusion levels for the 19 countries used in this research: Austria, Belgium, Canada, Denmark, Finland, France, Germany, Greece, Ireland, Italy, Netherlands, Norway, Portugal, Spain, Sweden, Switzerland, Turkey, UK, and USA) 
PC and Internet diffusion patterns for all countries in the dataset employed in our empirical analysis. While we examine the adoption of personal computers and the Internet here, the impact of complementary products is common to many high technology product categories (e.g., computers and software, streaming boxes and home theater systems, etc.), and, accordingly, our framework can be adopted to fit a number of similar industries/applications.

Empirically, we find convincing evidence for (a) the existence of asymmetric cross-product effects (asymmetric crossproduct effects exist when the size of the prior adopting pool for product $i$ has an impact on the growth of product $j$, but not the reverse), (b) differences in what is observed within versus across countries, and (c) a change in the composition of the network and cross-product effects over time that varies from country-to-country. More specifically, we are able to empirically disentangle the impact of (i) local network effects from (ii) foreign (cross-country) network effects from (iii) crossproduct effects on overall market potential and on incremental adopters. In doing so, we are able to decompose the relative contribution of each to the overall diffusion process on a country-by-county basis and see how each influence varies over time and across products, building on existing research on cross-country diffusion $[27,31]$ and time to takeoff [42].

\section{The Model}

We assume that consumer utility for adopting a technology is a function of several influences affecting the diffusion process, including the size of the prior adoption pool consisting of three components: within-country prior adoption, crosscountry prior adoption, and the prior adoption of complementary products. Accordingly, we extend the limited empirical research (see [36]) on cross-product network effects to assess cross-country effects in the spirit of Putsis et al. [27]. Since the size of each of the existing networks (within-country, crosscountry, and cross-product) will play an important role in our model and subsequent analysis, the role of network effects between two interacting technologies become central to our analysis. Consequently, we need to understand how the research issue at hand relates to the broader literature on network externalities. To this, we now turn.

\subsection{The Role of Direct Versus Indirect Networks}

Network externalities, first defined and discussed by Rohlfs [30], refers to the notion that the value of a product to one user depends in a systematic fashion on how many other users there are or, more specifically, the value of connecting to a network depends on the number of other people already connected to it (see $[10,20,32]$ for comprehensive early surveys). Recent research has made a careful distinction between direct versus indirect network effects (see [36]). In markets where direct network effects are present, the utility that consumers derive from acquiring an innovation is affected by the number of existing users or installed base of that innovation. This effect is present in a number of disparate technologies: the utility derived from adopting a mobile phone for text messaging or joining a social network is often positively related to the number of existing users. Indirect network effects exist when the utility of a product is related to the existing users of a compatible complementary product (see, e.g., $[3,36])$. In the presence of these cross-product interactions, the adoption level of one technology affects the speed of adoption of an interacting technology.

Despite the many sources of interaction across technologies presenting network effects, research on indirect network effects is limited. Notably, it has been conducted at the local level only (e.g., within a single country, see, e.g., [34, 40]). Furthermore, empirical analysis of indirect network effects is rare and limited to the analysis of the within-country relationship between software availability and hardware sales [34, 36]. Accordingly, our research builds on the limited empirical work conducted in this area by expanding prior research to examine network effects in a multi-country environment, empirically examining not only within-country but crosscountry effects.

2.2 Building a Cross-Country Diffusion Model with Indirect and Direct Effects with Varying Market Potential

Accordingly, in this research, we build a framework to model the cross-country diffusion process that possesses both direct and indirect effects, as well as covariates (e.g., price, country GDP, cultural variables). ${ }^{1}$ In such a model, the diffusion in one country should depend not only on the prior adopting pool within the home country, but upon the number of foreign users. For some products, the size of existing local users most affect individual utility, whereas the utility resulting from other applications may be dominated by foreign components for other products (e.g., the growth of global information content on the Internet). The relative impact will likely be idiosyncratic to the product category. Our general structure allows for the analysis of the diffusion of any possible set of interacting networks, from hardware-software complementary systems to compatible communication tools and enables us to

\footnotetext{
${ }^{1}$ Prior research in a diffusion setting has suggested that a larger existing network can increase utility vis-à-vis the larger network size, but it can also increase the impact of word-of-mouth. Here, our methodology and results relate to the impact of the size of the prior adopting pool irrespective of the source (word-of-mouth and/or network externality) and, accordingly, we will purposefully use the general terms (a) "direct" (i.e., own product), (b) "indirect" or "cross-product" (complementary product), and (c) "cross-country" or "foreign" effects throughout. Occasionally, when our discussions pertain to the size of the prior adopting pool, we will also use the general terms "size of network."
} 
dissect the foundation for diffusion in any one country, an important antecedent to multinational strategic development.

Thus, we build on prior work by incorporating an endogenous potential market that is allowed to vary across time and which captures within-product ("direct") and cross-product ("indirect") effects. In doing so, we consider the impact of these effects on the total size of the market on a country-bycountry basis; studies that allow for fluctuations in (potential) market size are rare. We also allow for these direct effects to have both a within-country ("local"), as well as a crosscountry ("foreign") component. As noted above, we use data from a set of interacting products (personal computers and the Internet) to assess how each effect shapes the diffusion process and the speed and dynamics of the adoption levels.

Consistent with early work in the diffusion of innovations literature [29], in particular for technology $x$ in country $i$, we consider a social system, $S_{x i}(t)$, within which an innovation diffuses. Only a certain part of the social system considers the intrinsic utility derived from the innovation to be sufficiently large to consider adopting it. A bounded variable, $0 \leq C_{x i}(t) \leq 1$, indicates the monotonically non-decreasing cumulative fraction of the social system susceptible to adoption at any time $t$. We define the potential market at any point of time, $M_{x i}(t)$, as the portion of the social system within which the innovation is eligible to diffuse as follows ${ }^{2}$ :

$M_{x i}(t)=C_{x i}(t) S_{x i}(t)$

In a number of product markets, the utility that consumers derive from acquiring an innovation is likely to be, at least in part, a function of the number of existing users. Hence, we extend the standard diffusion model for technological innovations by considering that for every period $t$, the proportion of the population susceptible to adoption, $C_{x i}(t)$, varies in a systematic fashion with the sizes of the existing adoption pool, an assumption that, we believe, is considerably more relevant to firm strategy in practice. The variable $N_{x i}(t)$ denotes the cumulative number of adopters of technology $x$ in country $i$ at time $t$. We assume that not only the local users but also the

\footnotetext{
$\overline{{ }^{2} \text { We can view }} C_{i}(t)$ as a "ceiling parameter," capturing the cumulative proportion of the social system "susceptible" to adopting the innovation at any point in time; see Putsis and Srinivasan [28] for a theoretical discussion of threshold utility. Putsis and Srinivasan [28] examine conditional indirect utility with and without purchase to investigate purchase deliberation, while Putsis et al. [27] uses the same framework to examine time varying diffusion parameters in a Bass-type model. Here, technically, $C_{i}(t)$ represents the proportion of a population for which conditional indirect utility with purchase is greater than without [27], which will change over time by definition. Accordingly, $\mathrm{M}_{i}(t)$ should not be interpreted as a static "eventual number of adopters" as in a standard Bass model. Rather, we advance this concept so that $\mathrm{M}_{i}(t)$ represents the market potential at time $t$, which should be expected to grow over time as additional individuals become susceptible as the size of the prior adopting network grows. Thus, $C_{i}(t), \mathrm{S}_{i}(t)$ and hence $\mathrm{M}_{i}(t)$ are all monotonically increasing in $t$, and $\mathrm{M}_{i}(t)$ should approach $M$ in a standard Bass model as $\mathrm{t} \rightarrow \infty$.
}

number of foreign users have an impact on the consumers' utility for adopting the technology. We therefore assume that $C_{x i}(t)$ depends in a systematic way on both the number of local, $N_{x i}(t)$, and foreign $\sum_{j \neq i} N_{x j}(t)$ users, and that the magnitudes of the effects present in local versus international markets are likely to be different for obvious reasons. We allow for indirect effects via an interacting technology $y$ : the size of the potential market $M_{x i}(t)$ also grows with the prior adoption level of the complementary product, denoted by $N_{y i}(t)$. Furthermore, the proportion of the social system willing to adopt the innovation, $C_{x i}(t)$, grows exponentially with the relevant prior adoption. We express both the potential market and the effective network sizes as fractions of the respective social systems to facilitate inter-product and inter-country comparisons. Thus, the potential market for country $i$ as a function of prior adoption levels becomes ${ }^{3}$ :

$$
\begin{aligned}
\frac{M_{x i}(t)}{S_{x i}(t)} & =C_{x i}(t) \\
& =1-\theta_{x} \exp \left[-\gamma_{x}\left(\frac{N_{x i}(t)}{S_{x i}(t)}\right)-\widetilde{\gamma}_{x}\left(\frac{\sum_{j \neq i} N_{x j}(t)}{\sum_{j \neq i} S_{x j}(t)}\right)-\widehat{\gamma}_{x y}\left(\frac{N_{y i}(t)}{S_{y i}(t)}\right)\right]
\end{aligned}
$$

where the parameters $\theta_{x}, \gamma_{x}, \widetilde{\gamma}_{x}$, and $\widehat{\gamma}_{x y}$ capture the shape of the growth of the potential market as a function of local, foreign, and complementary product prior adoption pools. We note that while we address complementary products here, i.e., we would expect the estimated $\widehat{\gamma}_{x y}$ to be greater than zero in the empirical application below, the degree of complementarity will strongly influence the estimated $\widehat{\gamma}_{x y}$ in practice. That is, we would expect strong complements to be close to +1 in value, unrelated products to be close to 0 , and substitute products close to -1 in value.

In the interest of keeping our framework as general as possible, we begin with a standard "Bass-type" diffusion approach by expressing the number of new adopters of the innovation $x$ by the following equation. This is a "baseline" model only-we will allow and test for alternative specifications in the next session. Also note that the basic framework that we set out can be extended to include most any cumulative distribution function (e.g., extending this framework to a

\footnotetext{
${ }^{3}$ Note that we include only "local" indirect effects here, but that the model can easily be extended to include an additional term to address cross-country indirect effects as well. One can think of a $2 \times 2$ matrix of indirect/direct and foreign/local effects. We extended and estimated the model developed here to include this fourth effect (foreign indirect effects) and found the gamma parameter to be statistically insignificant and the nested hypothesis test supported the nested model (one without the foreign effects present), suggesting that foreign indirect effects were not significant, at least not in the markets studied here. Accordingly, we present the parsimonious model that includes three out of four effects above. We note this here to point out that extending the model to include foreign indirect effects is a trivial extension that may be applicable in other empirical applications.
} 
generalized Bass model is straightforward). Furthermore, note that we assume temporally constant parameters in the model developed here in the interest of parsimony. We extend this basic framework below to allow the effects to vary over time and report the results and the implications for dynamic evolution over time there.

$n_{x i}(t)=\left[\alpha_{x i}+\beta_{x i} \frac{N_{x i}(t-1)}{M_{x i}(t-1)}\right]\left[M_{x i}(t-1)-N_{x i}(t-1)\right]$

For each country $i, n_{x i}(t)$ denotes the number of new adopters of innovation $x$ at period $t, N_{x i}(t)$ denotes the cumulative number of adopters of the innovation at the beginning of period $t, M_{x i}(t)$ denotes the potential market (as expressed in Eq. 2), $\alpha_{x i}$ is the "coefficient of external influence," and $\beta_{x i}$ is the "coefficient of internal influence" [1]. In this model, the portion of the social system willing to adopt an innovation is an increasing function of the relevant prior adoption pool. As a result, the role of external influence is likely to be smaller during the early stages of the diffusion process than it is in a standard Bass model, as we discuss in Section 4.3. We note that the varying impact of the network sizes could also help explain the findings of Van den Bulte and Joshi [41] regarding the time varying nature of the diffusion process.

\subsection{Incorporating Covariates}

The basic form of the dynamic diffusion process is governed by Eqs. (2) and (3). However, covariates clearly not only play a role in the takeoff process [42], but also in the post-takeoff diffusion path (e.g., [2, 24, 26, 38]). When we address crossproduct effects in the diffusion process, the role of tactical decisions (i.e., marketing mix variables) and economic and cultural covariates all become particularly important - a relative price decline in one product, for example, can play an important role in encouraging faster diffusion of the other. Hence, we need to not only account for cross-product interactions, but also incorporate variables that address crosscountry and cross-product heterogeneity into the framework developed (these can include a variety of marketing mix, economic and cultural variables - see [38, 42]).

In terms of the specific covariates to include in our model, we followed previous research closely $([27,38]$ and especially [42]). In the interest of parsimony and noting both potential collinearity and the complexity of our model, we rely on recent research by Gelder and Stremersch [12]. Specifically, a major conclusion drawn from Gelder and Stremersch [12] is that the dominant covariate in cross-national diffusion is economic in nature. Thus, we initially included the following covariates here: price, economic wealth (as captured by GDP per capita), and culture [17, 18]. We define price as changes in the price level $\left(\frac{p_{x i}(t)-p_{x i}(t-1)}{p_{x i}(t-1)}\right)$ and per capita gross domestic product in country $i$ at period $t, P C G D P_{i}(t)$, measured at purchasing power parity (1995 US) to allow for comparisons of the real purchasing power across countries (the variable was transformed by subtracting the average value for the entire sample for interpretation purposes).

We capture the cultural heterogeneity across countries by incorporating Hofstede's $[17,18]$ cultural variables in the model. Hofstede's dimensions are the most commonly used constructs for examining the effects of culture in marketing (e.g., $[11,22,25])$, as well as product adoption and diffusion rates between different countries [42] and related [19, 21, 22]. In the present application, we reduced the dimensionality of the cultural variables by employing factor analysis. The main factor, capturing $42 \%$ of the total cultural variance across the countries, is highly correlated with the first two Hofstede variables: power distance $(+0.86)$ and individualism $(-0.89)$. ${ }^{4}$ In our analysis, we use this main cultural factor as a third covariate, included in the model specification together with the marketing and economic covariates.

\section{Data and Estimation, Model Selection}

\subsection{Data and Estimation}

We estimate this model to determine the impact of (a) local direct effects, (b) foreign direct (cross-country) effects, and (c) indirect (cross-product) effects on the diffusion processes of home PCs and the Internet across 19 countries. We note that the methodology proposed here is applicable to any industry setting characterized by symmetric or asymmetric effects, multiple countries or segments, and the existence of interacting products; it also nests most standard diffusion models, including the Dekimpe et al. [7] model, making fit comparisons easier.

In estimating the model using data for the $\mathrm{PC}$ and the Internet, we use data that cover 19 countries in Europe and North America. Data on PC penetration - the number of households owning a PC-are available for the period 19812009. Data on penetration of a complementary product, the Internet — specifically, the number of home Internet users - are available for the period 1991-2009 (given that we are fortunate to have data captured very early in the industry's lifecycle, left censoring issues should not be an important concern here).

\footnotetext{
${ }^{4}$ The second factor captures an additional $26 \%$ of the total cultural variance across the countries. This factor-highly correlated with the other two Hofstede variables: masculinity $(+0.81)$ and uncertainty avoidance $(+0.74)$ - failed to be significant under any alternative specification, and hence has been discarded from our analysis. This is consistent with prior research-Yeniyurt and Townsend [43] find empirical evidence of the impact of power distance and individualism on the diffusion of three products while Steenkamp et al. [33] find strong evidence of individualism positively affecting the level of innovativeness.
} 
Consistent with the discussion earlier, we also obtained data on other relevant variables, such as the number of households, the total population size, the real per capita disposable income (more specifically the real per capita GDP measured at purchasing power parity, 1995 US\$), and an index of prices for all countries and for each year under investigation. We compiled the data using various Euromonitor reports, most notably its annual European Marketing Data and Statistics Report and World Marketing Data and Statistics Report. In line with our measures of diffusion of home PCs and the Internet, we use the total number of households and the total population size as measures of the respective social systems. We use real per capita national product and prices as explanatory covariates for the diffusion of home PCs. For the cultural variables, we used the Hofstede measures - originally established by Hofstede [17, 18] — as discussed above.

For estimation, we used data through 2005, leaving the remaining period (2006-2009) as a holdout sample. We estimated Eqs. 2 and 3 using full information maximum likelihood (FIML) to account for the endogeneity of the regressors [15]. We assess the fit by calculating a system-wide measure of adjusted $R^{2}$, using a system-wide sum of squared errors (SSE). Noteworthy, the proposed structure nests the Dekimpe et al. [7] model as the particular case with no network effects, where the potential market is a constant fraction $(1-\theta)$ of the social system. Therefore, we additionally estimate a restricted model without network size effects $\left(\gamma_{x}=\widetilde{\gamma}_{x}=\widehat{\gamma}_{x y}=\gamma_{y}=\widetilde{\gamma}_{y}=\widehat{\gamma}_{x y}=0\right)$. We employ a standard log-likelihood ratio test to determine the significance of the impact of the size of the network.

\subsection{Model Selection}

We begin the analysis by testing the significance of the network structure and the inclusion of covariates considered in our proposed framework: we estimated the more general (full) model as well as two nested specifications: one without network effects and one without covariates. ${ }^{5}$

\footnotetext{
${ }^{5}$ We note that while Eqs. (2) and (3) govern the dynamics of the model, there are at least three possibilities for the ways in which covariates can enter Eq. (3): (a) covariates enter linearly via the coefficient of external influence, $\alpha_{x i}$, (b) covariates enter linearly via the coefficient of internal influence, $\beta_{x i}$ in Eq. 3, or (c) covariates enter in a multiplicative fashion via a generalized Bass structure. We spent a great deal of time investigating which of the three specifications fit best-we preferred to let the data dictate which approach is most appropriate given the current empirical application. Here, no one of the three specifications dominated non-nested Vuong tests. However, specification (b), with the covariates entering linearly via the coefficient of internal influence, $\beta_{x i}$ in Eq. 3, produced results consistent with expectations and of appropriate sign and statistical significance for key variables. Thus, specification (b) seems to be a reasonable one to proceed with, although we note that the answer to which covariate specification is most appropriate may be context-specific.
}

Overall, the results strongly support the inclusion of both network effects and covariates: the LL ratio test for the full model versus the restricted model without network effects was $439.8\left(\mathrm{PR}\left(H_{0}=\right.\right.$ restricted model $)=1.45 \mathrm{e}-66 \mathrm{w} / 44$ additional variables) and the LL ratio test for the restricted model without covariates was $33.68\left(\mathrm{PR}\left(H_{0}=\right.\right.$ restricted model $)=2.76 \mathrm{e}-6 \mathrm{w} /$ 5 additional variables). Accordingly, we proceed with the full model below.

Identification is achieved through the prior adoption variables inside of the non-linear functional form (that is each $\gamma$ is identified by its analogous prior adoption pool). In order to ensure that identification and parameter recovery is possible, a series of 10 simulations generating 530 estimated parameters was conducted. As should be expected, in $95.85 \%$ of the comparisons, the original parameters lied within the $95 \%$ confidence interval (508 out of 530 estimated parameters), confirming our ability to identify and recover the underlying "true" parameters.

Finally, we compared both the level of fit and the predictive ability to a set of alternative competing adoption models. The first alternative specification [27] accounts for cross-country effects but does not consider cross-product interactions. The second model [36] captures the indirect network effects (hardware-software interaction) without considering crosscountry influences. To make this model fully comparable to our specification, we use new adoptions for home PCs instead of "hardware sales," and the Internet-user base instead of the "software availability." The third model is a nested version of our model that does not include network effects [7]. We estimated all models using time series through 2005 , leaving the remaining period (2006-2009) as a holdout sample, comparing both withinsample fit and fit into short- and longer-term forecast periods. The proposed model with network effects and covariates entering linearly via the coefficient of internal influence dominates withinsample and holdout sample fit versus competing models.

\section{Results and Analysis}

In order to put our discussion of the results in proper perspective, we proceed as follows:

Dynamic Implications of Our Proposed Model Before we interpret the empirical results directly, we present graphs from simulations that help to understand the dynamics of the proposed model, which sets the stage for a more meaningful discussion of the results. In addition, we extend the model to include time-varying indirect effects and explore the implications of doing so.

Understanding the Big Picture-Strategic Market Considerations We begin our discussion of the empirical results by discussing the overall "picture" that the results paint - the results should have face validity. Since 
documenting and understanding the additional strategic insight our approach provides is the key objective of this research, we address the key insights for PCs and the Internet here and address the broader implications of the insight gained in the paper's final section.

Decomposing the Impact of Local Direct, Foreign Direct, and Indirect Effects We decompose the impact of each of the three effects on overall market potential and incremental adoptions over time, for each country and each period.

\subsection{Dynamic Implications of the Proposed Model}

The dynamic interaction across the three prior adoption pools (within-country, foreign (cross-country), and cross-product) has important strategic implications for firms: the three adopting populations interact in a complex fashion to affect both the speed of adoption and the overall market potential.

In order to illustrate the dynamics of the diffusion process in our model, we analyze the partial effect of the prior adoption level $N_{x i}(t) / S_{x i}(t)$ on the diffusion process (the local direct effect) via a series of simulations. Figure 2 illustrates the potential market size and the adoption processes for hypothetical fixed values of $\alpha_{x}$ and $\beta_{x}$ (setting $\alpha_{x}=0.03$ and $\beta_{x}=0.38$, average values reported by [37] in their meta-analysis), and various combinations of $\gamma_{x}$ and $\theta_{x}$. The initial adoption level at period 1 was set to $0.1 \%$ and all combinations of the parameters $\gamma_{x}$ and $\theta_{x}$ present the same long-term adoption level of $90 \%$. The intent of these graphs is to demonstrate how for fixed values of $\alpha_{x}$ and $\beta_{x}$ and the same ultimate long-term adoption level, different values of $\theta_{x}$ and $\gamma_{x}$ give rise to very different diffusion patterns. Conceptually, we note that any decision or factor that, ceteris paribus, can speed up the diffusion process, the better it is for the NPV of the launch. Thus, the dynamics of these relationships can have profound implications on the firm's strategy.

To illustrate, Fig. 2 demonstrates this clearly - we can see that different combinations of parameters $\theta_{x}$ and $\gamma_{x}$ indeed give rise to very different diffusion patterns (the top panel presents potential market size as a function of the adoption level, the middle panel presents the new adopter dynamics, and the bottom panel presents the overall adoption dynamics across $t)$. The marginal contribution, $N_{x i}(t)$, to the growing potential market, $M_{x i}(t)$, is positively related to $\gamma_{x}$-for higher (lower) values of $\gamma_{x}$, the impact of the size of the prior adoption pool becomes relatively more (less) important and the potential market $M_{x i}(t)$ grows more (less) quickly, approaching a higher (lower) fraction of the social system $S_{x i}(t)$. By comparison, for $\gamma_{x}=0$, our model converges to Dekimpe et al. [7] with an endogenous, but constant potential market - a potential market that is a constant fraction $(1-\theta)$ of the social system.

Conceptually, it is important to note that these graphs illustrate that diffusion patterns in a model with strong direct product effects differ significantly from those in a regular Bass model: adoption is slower in the early stages, but increases relatively rapidly once a threshold level of adopters has been reached. The larger the size of the prior adopting pool, the later the diffusion process peaks. These results are important since the model developed here captures very different and more general diffusion patterns. For example, later in the paper, we will partition total diffusion into the three diffusion components (local, foreign, and cross-product) - this combined with the simulation results in Fig. 2 will shed substantive light on both how these markets evolve and how managers should "handle" the diffusion process. We will draw some conclusions regarding these managerial implications in the final section of the paper.

One important point from the graphs below is that the potential market $C_{i x}(t)$ in Eq. (2) increases with the size of the network and grows at a decreasing marginal rate with the size of any of the interacting networks - higher values of $\theta(0<\theta<1)$ go handin-hand with a smaller potential market size, and therefore a slower diffusion process. That is, the lower the number of initial adopters and/or the smaller fraction of the social system unaffected by overall size effects $(1-\theta)$, the longer it takes for diffusion to take off and the lower the long-run adoption level. Noteworthy, the elasticity of the potential market, $M_{x i}(t)$, with respect to the size of any of the three interacting prior adopting pools-local, external, and indirect-is proportional to the
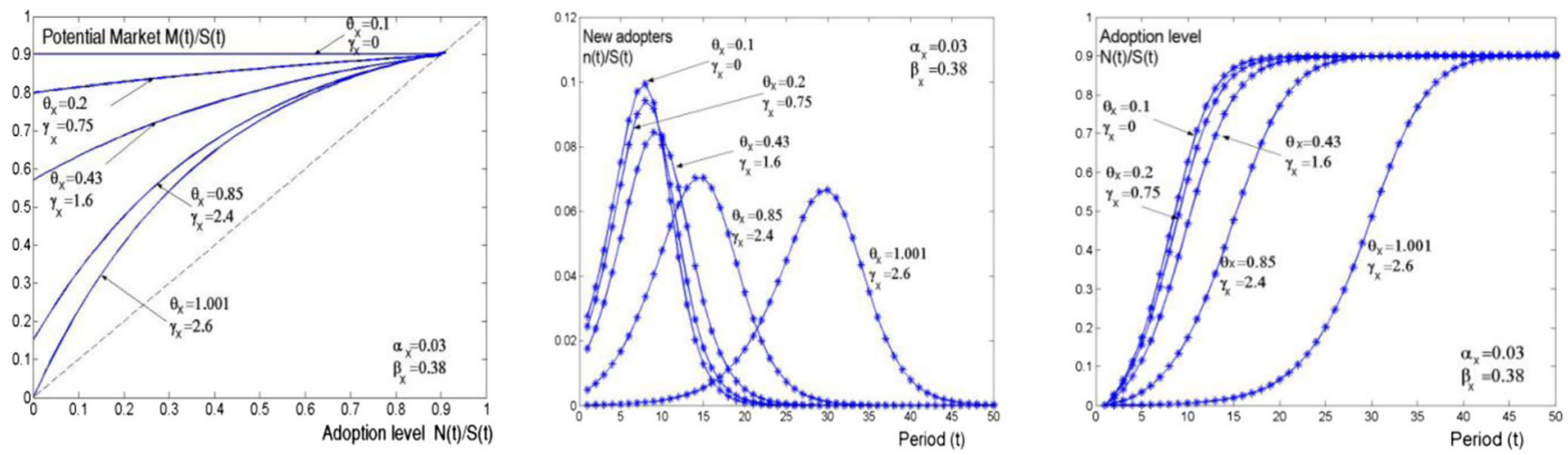

Fig. 2 Potential market size, new adopters, and adoption levels for different values of $\gamma_{x}$ 
respective size of the network effect parameters $\gamma_{x}, \widetilde{\gamma}_{x}$, and $\widehat{\gamma}_{x y}$, which provide estimates of the strength and existence of the individual network size effects (a parameter $\gamma=0$ implies the absence of the corresponding network size effect).

Conceptually, this provides an alternative explanation to the threshold model of Goldenberg et al. [13] in that the (continuous) endogenous growth of the potential market can explain the slow growth and takeoff (the common "hockey stick") often observed - overall growth depends in a complex fashion on the local direct market, the foreign direct market, the network for relevant complementary products, the magnitude of the three network parameters, the inherent strength of the diffusion process itself (captured here by $\alpha$ and $\beta$ ), as well as the relevant covariates (such as price, GDP, and culture). The diversity of diffusion paths that this process will accommodate is significant, an important part of multi-faceted international diffusion patterns in reality, and an important part of launch strategy decisions in a multinational setting.

We also extended the framework to allow for variation in the indirect effects over time. The diffusion of home PCs started in 1981, reaching an aggregate adoption level across the sample of countries studied of $13 \%$ (20\% in the UK and $16 \%$ in the USA and Canada) in 1991 (when the diffusion of the Internet started). Differences in penetration rates across countries and over time may very well have produced crossproduct interactions that varied across time. For instance, we would expect that the installed base of home PCs might have had a significant, positive, and growing impact on the diffusion of the Internet during the early years of the Internet, but that this impact may have decreased or even reversed later in the sample. Thus, we expand the framework presented thus far to allow for dynamic cross-product interactions assuming that, for both technologies, the cross-product effect varies over time according to $\hat{\gamma}(t)=\hat{\gamma}_{0} \quad 0.5^{\left(\phi|\phi|^{(t-1992)}\right)}$ —using an exponential specification allows for cross-product effects to evolve over time according to the magnitude of the parameter $\phi$. For example, when $\phi=0$, the effect is constant over time. Figure 3 illustrates the potential effect of $\phi$ on the evolution of $\widehat{\gamma}(t)$ over time. For positive values of $\phi$, the effect grows over time, while for negative values of $\phi$, the effect dissipates.

The higher the absolute value of the parameter, the greater the effect varies across time. In the long run, the magnitude of the effect converges to $\hat{\gamma}_{0}$, precisely as one would expect.

\subsection{Understanding the Big Picture-Strategic Market Considerations}

We present the overall results in Table 1 below and begin with the estimated results for PCs, turning our attention to the Internet after. The estimated local direct effect parameters for home PCs are statistically significant and high in magnitude, ranging between 0.27 and 1.32 for all countries but Turkey and

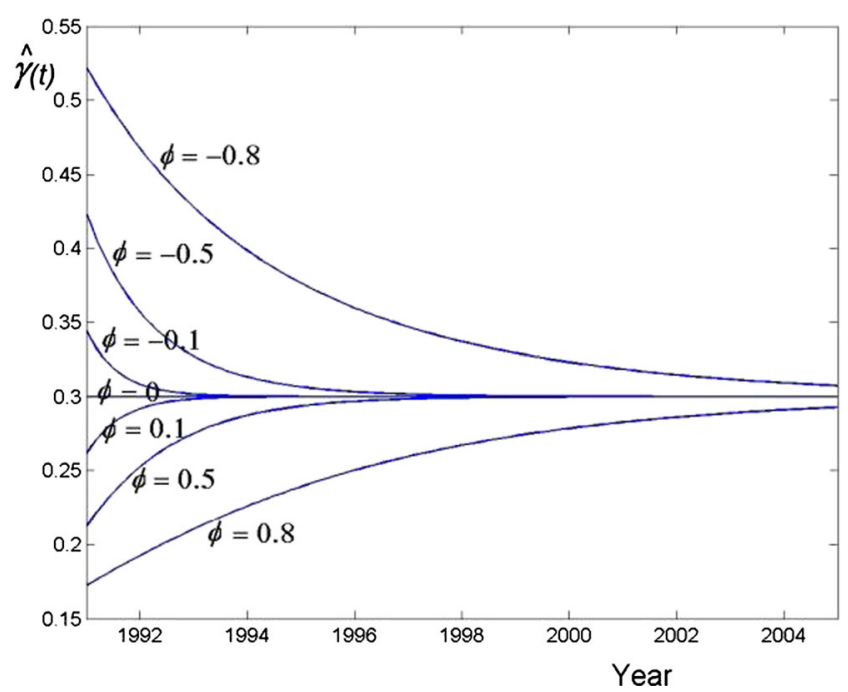

Fig. 3 Effect of $\phi$ on the evolution of $\widehat{\gamma}(t)$ on time $\left(\hat{\gamma}_{0}=0.3\right)$

Portugal. In general, these estimates reveal dramatic local direct effects where the willingness to adopt the product evolves with the number of previous local adopters. Simply stated, adoption of PC's is predominantly a local phenomenon - we see friends and co-workers on PCs, which encourages adoption at the local level. Despite the significance and high magnitude of the local direct effect parameter $\gamma_{x}$, foreign adoption of PCs is found to have a significant but small impact on local adoption $\left(\widetilde{\gamma}_{x}=\right.$ 0.093). In addition, the cross-product effect of prior Internet adoption (starting in 1991) on PC sales is relatively small in the first few years - the cross-product effect of prior Internet adoption only begins to have an impact on the diffusion of home PCs in the late 1990s for most countries.

When viewed in aggregate, these results suggest that the potential market for home PCs was primarily driven by the growth PC adoption in the focal (local) country, consistent with the prior results of Goolsbee and Klenow [14]. This is not at all surprising, supporting our contention that the results overall have strong face validity. In contrast, all three estimated effects are positive and relevant for the Internet. The local direct NE parameter is significant at a $5 \%$ level $\left(\gamma_{y}=0.181\right)$ the cross-country adoption level varies significantly across countries, with relatively high values such as 1.84 and 1.28 for Sweden and Finland versus nonsignificant values for Turkey and Ireland (see Table 1).

As noted earlier, and as shown in Fig. 4 below, we also plotted the various effects over time. ${ }^{6}$ The estimated indirect effect of PC adoption Internet diffusion $\widetilde{\gamma}_{y x}$ starts at 0.578 , and evolves over time, converging to a long-term value of 1.158. This is precisely the opposite of the cross-product effects for Home PCs: in the early stages of Internet development (e.g., 1991), the aggregate adoption level of Home PCs for the 19

\footnotetext{
${ }^{6}$ A complete set of country decompositions over time for all countries in the sample is available as supplemental material available online.
} 
Table 1 Proposed model parameter estimates

\begin{tabular}{|c|c|c|c|c|c|c|}
\hline \multirow[t]{2}{*}{$R^{2}$} & \multicolumn{3}{|c|}{ Home PCs } & \multicolumn{3}{|c|}{ Internet } \\
\hline & \multicolumn{3}{|c|}{0.9453} & \multicolumn{3}{|c|}{0.8955} \\
\hline Coefficient of external influence & $\alpha_{x}$ & 0.013 & $(0.087)$ & $\alpha_{y}$ & 0.017 & $(0.018)$ \\
\hline Coefficient of internal influence & $\beta_{x}$ & 0.648 & $(0.152)$ & $\beta_{y}$ & 0.923 & $(0.172)$ \\
\hline Early-adopter parameter & $\theta_{x}$ & 0.992 & $(0.002)$ & $\theta_{y}$ & 1.017 & $(0.007)$ \\
\hline \multicolumn{7}{|l|}{ Effects: } \\
\hline \multirow[t]{19}{*}{ 1. Local (varying by country) } & \multirow{19}{*}{$\gamma_{x}$} & Austria & $0.81(0.25)$ & \multirow[t]{19}{*}{$\gamma_{y}$} & \multirow[t]{19}{*}{0.181} & \multirow[t]{19}{*}{$(0.082)$} \\
\hline & & Belgium & $0.95(0.32)$ & & & \\
\hline & & Canada & $0.97(0.08)$ & & & \\
\hline & & Denmark & $1.32(0.35)$ & & & \\
\hline & & Finland & $0.71(0.21)$ & & & \\
\hline & & France & $0.60(0.11)$ & & & \\
\hline & & Germany & $0.95(0.04)$ & & & \\
\hline & & Greece & $0.27(0.13)$ & & & \\
\hline & & Ireland & $0.68(0.11)$ & & & \\
\hline & & Italy & $0.46(0.07)$ & & & \\
\hline & & Netherlands & $1.19(0.16)$ & & & \\
\hline & & Norway & $1.01(0.82)$ & & & \\
\hline & & Portugal & $-0.71(1.70)$ & & & \\
\hline & & Spain & $0.70(0.17)$ & & & \\
\hline & & Sweden & $1.27(0.44)$ & & & \\
\hline & & Switzerland & $1.05(0.49)$ & & & \\
\hline & & Turkey & $-0.23(0.53)$ & & & \\
\hline & & UK & $0.76(0.04)$ & & & \\
\hline & & USA & $0.78(0.04)$ & & & \\
\hline \multirow[t]{19}{*}{ 2. Cross-country } & \multirow[t]{19}{*}{$\widetilde{\gamma}_{x}$} & \multirow[t]{19}{*}{0.093} & \multirow[t]{19}{*}{$(0.012)$} & \multirow[t]{19}{*}{$\tilde{\gamma}_{y}$} & Austria & $1.19(0.54)$ \\
\hline & & & & & Belgium & $0.30(0.07)$ \\
\hline & & & & & Canada & $072(0.12)$ \\
\hline & & & & & Denmark & $1.15(0.05)$ \\
\hline & & & & & Finland & $1.28(0.45)$ \\
\hline & & & & & France & $0.18(0.05)$ \\
\hline & & & & & Germany & $0.54(0.03)$ \\
\hline & & & & & Greece & $0.04(0.15)$ \\
\hline & & & & & Ireland & $-0.09(0.27)$ \\
\hline & & & & & Italy & $0.31(0.07)$ \\
\hline & & & & & Netherlands & $1.05(0.61)$ \\
\hline & & & & & Norway & $1.05(0.03)$ \\
\hline & & & & & Portugal & $0.37(0.11)$ \\
\hline & & & & & Spain & $0.35(0.12)$ \\
\hline & & & & & Sweden & $1.84(0.43)$ \\
\hline & & & & & Switzerland & $0.71(0.16)$ \\
\hline & & & & & Turkey & $-0.09(0.15)$ \\
\hline & & & & & UK & $0.79(0.07)$ \\
\hline & & & & & USA & $0.91(0.03)$ \\
\hline \multirow[t]{2}{*}{ 3. Cross-product function $\widehat{\gamma}(t)$} & $\widehat{\gamma}_{x y}$ & 0.562 & $(0.013)$ & $\widehat{\gamma}_{y x}$ & 1.158 & $(0.125)$ \\
\hline & $\varphi_{x y}$ & -0.719 & $(0.007)$ & $\varphi_{y x}$ & 0.915 & $(0.056)$ \\
\hline \multicolumn{7}{|l|}{ Covariates } \\
\hline GDP & & $1.5 \mathrm{e}-5$ & $(4.91 \mathrm{e}-6)$ & & \multirow[t]{2}{*}{$4.4 \mathrm{e}-5$} & \multirow[t]{2}{*}{$(6.81 \mathrm{e}-6)$} \\
\hline Price & & 4.815 & $(5.626)$ & & & \\
\hline Cultural & & -0.588 & $(0.152)$ & & -0.297 & $(0.129)$ \\
\hline
\end{tabular}

Note: standard errors in parenthesis 


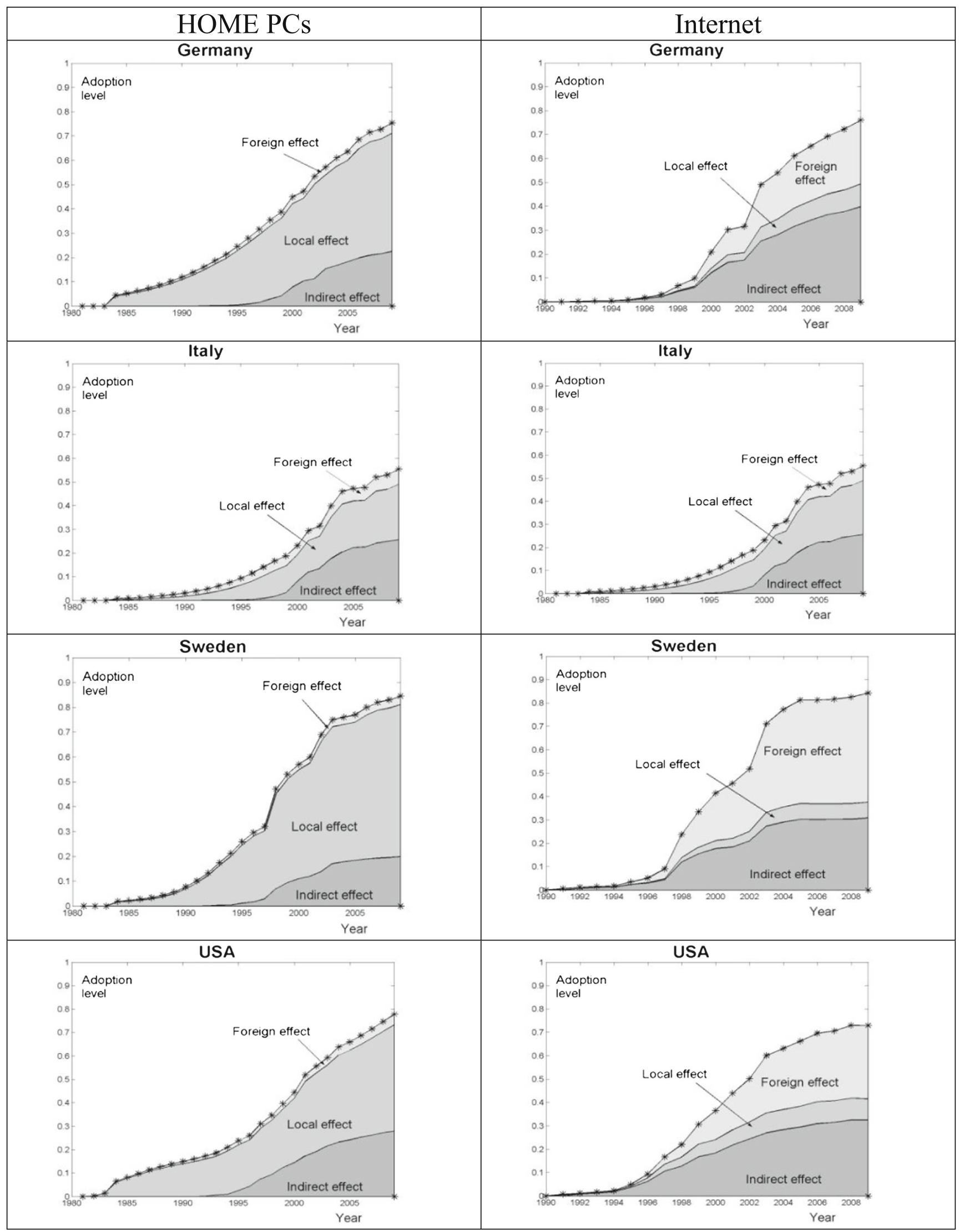

Fig. 4 Incremental adoptions for home PCs and the Internet (representative set; full set available as supplemental material online) 
countries studied was $12.6 \%$, with some leading countries such as the Netherlands and the UK having adoption levels of 22 and $20.4 \%$, respectively. As it will be seen below, the established adoption levels of Home PCs was the main driver of the diffusion of the Internet during its first years, contrasting sharply with the results for the PC market which had little crossproduct impact early on (again, not surprisingly).

These results reveal the global character of the Internet. Noteworthy, for the Internet, the fraction of early adopters is small in magnitude. This implies something not seen in other studies not addressing all three impacts - a large effect (local, foreign, or indirect) is required for ultimate success of the innovation - this effect can come from any of the three sources (again, local, foreign, or indirect). This is important, as there are substantive implications for the source of growth early on in the product lifecycle and is characteristic of any product presenting both significant direct and indirect effects from a complementary technology. Strategically, the order of entry is obviously deeply impacted by the origins of growth over the evolution of the diffusion process over time; such a finding can only be uncovered by examining all three effects together.

Therefore, we suggest that the complex nature of the three effects play a crucial role here, and this highlights the strength of the methodology employed and the related contribution:

- Home PC diffusion was largely driven by external influence led by local direct effects - the more individuals saw the penetration of home PCs grow locally, the greater the likelihood of adoption. This varied significantly across countries. Logically, we would argue that many hardware innovations, particularly those with a high price tag such as a PC, require seeing neighbors with the product and actually being able to touch and feel the product. Thus, we would naturally expect that local effects dominate and that the power of local versus foreign versus cross-product effects would vary significantly across countries.

- By contrast, Internet adoption was driven by a combination of influences: (a) Local Direct Effects (e.g., "I want to e-mail my friends"), (b) Foreign Direct Effects (e.g., "I need to access information from throughout the world," "The larger the set of users and information/sites available, the greater the benefit that I see"), and (c) Indirect Effects (e.g., "The greater the number of friends/family/colleagues with personal computers, the greater the benefit from social networks and the like"). Thus, we would logically expect the types of effects present in the case of "hardware" innovations (such as PCs) to be substantively different from "software" innovations (such as the Internet) that rely heavily on local hardware networks and global content development and delivery-and this is indeed what we find empirically.

- We would argue that, when taken in aggregate, these results highlight the importance of incorporating and estimating multi-market effects. By examining not only standard internal, external, and covariate (e.g., price, GDP, cultural variables) coefficients, but also estimating complementary product influences, one is able to obtain a significantly more complete view of the diffusion process in markets that develop and evolve dynamically over time. Clearly, this is the case with respect to the diffusion of PCs and the Internet.

- Conceptually, it is important to assess the relative strengths of each of the various effects studied here. Our empirical results highlight how one can examine these individual effects. In order to illustrate this, we now turn our attention to an illustration, examining the countryspecific sources of the each of the effects addressed here, in the spirit of Putsis et al. [27].

4.3 Decomposing the Impact of Local Direct, Foreign Direct, and Indirect Effects

In one related study, Goolsbee and Klenow [14], employing a database on the computer ownership and purchase decisions of US households, find that people are more likely to buy their first home computer in areas where a high fraction of households already own computers or when a large share of their friends and family own computers (i.e., the effect is local in nature). Opposite to the local character of the diffusion of home PCs, the potential market for the Internet is propelled by three different effects: local direct, foreign direct (crosscountry), and indirect (complementary or "cross-product"), as captured by the Eqs. (2) and (3). In order to better understand the contribution of these three drivers along the diffusion process at a country level, we decompose the marginal effects of each: local direct $\left(\frac{N_{y i}(t)}{S_{y i}(t)}\right)$, foreign direct $\left(\frac{\sum_{j \neq i} N_{y j}(t)}{\sum_{j \neq i} S_{y j}(t)}\right)$, and indirect $\left(\frac{N_{x i}(t)}{S_{x i}(t)}\right)$, for every country for each period. Taking partial derivatives in Eq. (3), we can decompose the incremental potential market $\left[M_{y i}(t)-M_{y i}(t-1)\right]$ as the sum of the partial derivatives times the growth in the size of the respective prior adopting pool:

$$
\begin{aligned}
\frac{M_{y i}(t)}{S_{y i}(t)}-\frac{M_{y i}(t-1)}{S_{y i}(t-1)}= & \gamma_{y} \overbrace{\left[1-\frac{M_{y i}(t-1)}{S_{y i}(t-1)}\right]\left(\frac{N_{y i}(t)}{S_{y i}(t)}-\frac{N_{y i}(t-1)}{S_{y i}(t-1)}\right)}^{\text {Local direct effects }} \\
& +\widetilde{\gamma}_{y} \underbrace{\left[1-\frac{M_{y i}(t-1)}{S_{y i}(t-1)}\right]\left(\frac{\sum_{j \neq i} N_{y j}(t)}{\sum_{j \neq i} S_{y j}(t)}-\frac{\sum_{j \neq i} N_{y j}(t-1)}{\sum_{j \neq i} S_{y j}(t-1)}\right)}_{\text {Foreign direct effects }} \\
& +\widehat{\gamma}_{y x} \underbrace{\left[1-\frac{M_{y i}(t-1)}{S_{y i}(t-1)}\right]\left(\frac{N_{x i}(t)}{S_{x i}(t)}-\frac{N_{x i}(t-1)}{S_{x i}(t-1)}\right)}_{\text {Indirect effects }}
\end{aligned}
$$


Thus, for every country and for each period, we can determine the fraction of the potential market growth $\left[M_{y i}(t) / S_{y i}(t)-M_{y i}(t-1) / S_{y i}(t-1)\right]$ originated by the evolution of each of the three types of effects. Using this equation year by year, we can determine, for each country, how each of the three effects contributes to the growth dynamics of the potential market, $M_{y}(t)$, from 1991 to 2009. Denote $M_{y}{ }^{l}(t), M_{y}{ }^{2}(t)$, and $M_{y}{ }^{3}(t)$ as the fractions of the potential market generated by the evolution of size of each of the three networks: local, foreign, and indirect, respectively, we divide the number of new adopters, $n_{y}(t)$, generated each period by the size of each of the three networks. As illustrated by the dynamics from Eq. (3), the new adopters $n_{y}(t)$ are derived from the fraction of the potential market that has not adopted the innovation, $M_{y}(t-1)-N_{y}(t-1)$ :

$n_{y i}(t)=\left[\alpha_{y}+\beta_{y} \frac{N_{y i}(t-1)}{M_{y i}(t-1)}\right]\left[M_{y i}(t-1)-N_{y i}(t-1)\right]$

Noting that for every period $t$, the adoption process in country $i$ occurs from within the market base $M_{y i}(t-$ 1) $-N_{y i}(t-1)$, we assume that the fraction of new adopters generated by the evolution of any of the three, denoted by $n_{y}{ }^{l}(t), n_{y}{ }^{2}(t)$, and $n_{y}{ }^{3}(t)$, is proportional to the size of its segments of potential adopters, $M_{y}{ }^{l}(t-1)-N_{y}{ }^{l}(t-1)$, $M_{y}{ }^{2}(t-1)-N_{y}{ }^{2}(t-1)$, and $M_{y}{ }^{3}(t-1)-N_{y}{ }^{3}(t-1)$, respectively. From this, for each period, we can divide new adopters, $n_{y}(t)$, the market potential, $M_{y}(t)$, and the cumulative number of adopters, $N_{y}(t)$, in the three segments as follows:

$N^{j}{ }_{y i}(t)=N^{j}{ }_{y i}(t-1)+n^{j}{ }_{y i}(t), \quad J=1,2,3$

where

$n_{y i}^{j}(t)=n_{y i}(t) \frac{M_{y i}^{j}(t-1)-N^{j}{ }_{y i}(t-1)}{\sum_{j=1}^{3} M^{j}{ }_{y i}(t-1)-N^{j}{ }_{y i}(t-1)}, j=1,2,3$

Iterating period by period in the previous equation system, we generate the time series for the cumulative number of adopters in country $i$ coming from each source beginning in period 1 .

The adoption levels generated by the three prior adoption types are plotted in Fig. 4 for both technologies across a representative subset of countries (all 19× 2 graphs, two graphs for each country in the sample, is available as supplemental material online). Table 1 combined with Fig. 4 reveal a number of interesting observations:

- The diffusion of home PCs is mainly driven by the growth of the local network. The cross-county effect is virtually inexistent for most countries. Only the lagged countriesGreece, Italy, Portugal, and Turkey—present a slower diffusion process with a relatively higher cross-country effect due to the limited local effect (see Table 1). Finally, the indirect effect is smaller, only starting to have a significant impact in the late 90 s.

- Thus, if one is launching a product such as a PC before network externalities kick in, launching in highly independent countries such as Sweden makes strategic sense.

- As shown in Table 1, the home-PC local-effect parameter is significantly lower for Turkey and Portugal than for the rest of countries. Therefore, these two countries present a slower diffusion process where the impact of the Internet on the diffusion of home PCs (indirect effect) is relatively higher than for the rest of countries. Noteworthy, Turkey and Portugal have adoption levels for home PCs significantly lower than the ones presented for the rest of countries during the period studied. In 2009, the adoption level of home PCs for Portugal and Turkey is 51 and $36 \%$, respectively, far below the aggregate adoption level for the set of countries, $71 \%$. For both countries, our model forecasts fit the underlying data well, suggesting that we are recovering real and important differences.

- Opposite to the local character of the evolution of home PCs, the potential market for the Internet is propelled by all three effects: local direct, foreign direct (cross-country), and indirect (complementary or "cross-product").

- For all countries, the adoption level early on is mainly determined by the complementary (indirect) product effect, as the prior direct product network size is still quite small, due to relatively low Internet adoption. This is consistent with observations made earlier that the early adoption level of home PCs drove early Internet adoption.

- As the adoption of the Internet grows, both direct effects (local and foreign) become relatively more important. This is shown by the flatter evolution of the Indirect effect compared to the direct ones. This is an intuitive result, as the adoption of the Internet has grown faster than the home PCs (see Fig. 1).

- For the Internet, the foreign effect is relatively high for most developed countries. The relative impact of this effect is determined by the size of the foreign-effect parameter (see Table 1). Particularly, five developed countries-Netherlands, Norway, Denmark, Finland, and Sweden - present the highest relative cross-country effect. On the other side, for the countries with lowest foreign-effect parameters - Turkey, Ireland, and Greece - the direct effect is mostly local. 
- Strategic launch decisions that focus on the high crosscountry impact of countries like Netherlands, Norway, Denmark, Finland, and Sweden will have the greatest likelihood of success in the long run and the quickest path to diffusion in other countries as well. Uniform ("sprinkler") strategies are likely to be relatively ineffective as a result.

\section{Conclusions, Strategic Implications, and Future Research Opportunities}

\subsection{Conclusions and Strategic Implications}

In this study, we demonstrate how diffusion models can be extended to incorporate both the complementary nature of two goods and related size of network effects within each of the two product categories. To our knowledge, this represents the first attempt in the strategy, marketing, and/or economics literature to incorporate both influences into a single framework.

In addition to the insights gained by incorporating network and cross-country effects, our proposed model produced estimates consistent with previous research - the estimates for the coefficient of external influence was 0.013 (home PCs) and 0.017 (the Internet), while the estimate for the coefficient of internal influence was 0.648 (PCs) and 0.923 (the Internet). These are consistent with the values reported by Sultan et al. [37], where the authors conducted a meta-analysis of previous diffusion models (213 sets of parameters from 15 articles), reporting values ranging from 0.000021 to 0.03297 for the external coefficient values ranging from 0.2013 to 1.67 for the coefficient of internal influence. Furthermore, we note that (a) the coefficient of internal influence is higher for the Internet than for PCs (0.923 versus 0.648), and (b) on average, the three sigma parameters (local + cross-country + cross-product) are significantly higher for the Internet than for the home PCs. Thus, in addition to recovering "standard" diffusion parameters, we are able to obtain estimates of the impact of other key factors such as cross-product and cross-country effects.

Several substantive results of our empirical analysis stand out. First, as expected, we find that home PC diffusion was driven predominantly by local direct effects - the more individuals saw the penetration of home PCs grow locally, the greater the likelihood of adoption. Second, by contrast, we find very different effects for the Internet-Internet adoption was driven by a combination of influences: (a) local direct effects, (b) foreign direct effects, and (c) indirect effects. These results are in line with the idea that the diffusion of one product can facilitate the diffusion of another product, but that the impact can be asymmetric across products. We would logically expect the types of effects present in the case of "hardware" innovations (such as PCs) to be substantively different from "software" innovations (e.g., the Internet) that rely heavily on local hardware networks and global content development and delivery. By examining not only standard internal, external, and covariate (e.g., price, GDP, culture) coefficients, but also estimating competing network influences, one is able to obtain a significantly more complete view of the diffusion process in markets that evolve over time.

Our methodology is applicable to any industry setting that involves interacting products or product categories, is characterized by size of network effects, and consists of multiple countries (or segments), following Bayus et al. [5]. Although conventional models can capture diffusion patterns presented by complementary innovations with size of network effects, a key feature of our nested modeling structure is that it explicitly selects the appropriate functional form. This allows for a better understanding of how potential markets evolve over time, as well as map the way in which the diffusion of an innovation affects and is affected by the diffusion of a complementary innovation.

More generally, armed with a better understanding of market dynamics, firms can aim to actively influence growth. For example, a grasp of how complementarities and network effects - within and across countries - affect the diffusion speeds and long-run market potentials can help firms in analyzing which markets to enter, in what order, and in what fashion. As noted above, here, strategic launch decisions that focus on the high cross-country impact of countries like Netherlands, Norway, Denmark, Finland, and Sweden will have the greatest likelihood of success in the long run and the quickest path to diffusion in other countries as well. Uniform ("sprinkler") strategies are likely to be relatively ineffective as a result, suggesting that sprinkler strategies will only be effective in situations where there is little interaction across adopting pools. Any entry decision should focus on countries with larger early adoption pools in markets where foreign adoption is important (e.g., launching in the UK or US early would have an impact in a country like Denmark, but would have little of no impact in Switzerland).

\subsection{Future Research Opportunities}

We recognize the limitations inherent in incorporating a complex set of effects: one cannot model everything. Consequently, there are at least four ways to extend this research. First, an effort can be made to incorporate the effect of additional marketing mix variables including country covariates that address socioeconomic differences across countries. Second, exploring a multi-product setting would be particularly interesting - given additional data on other related is certainly feasible. Third, we model the phenomena at hand as though 
we have a complete economic and cultural system- the impact of controlling for penetration outside of these 19 countries may be subject of future study. Finally, we think it is worthwhile to test our model for other complementary product combinations, either related to the products studied here or concerning other technologies (e.g., DVD players and discs, video games, and consoles). Such analyses should further advance our understanding of multi-product interactions and network effects in an international context.

Acknowledgments This research builds on earlier work conducted with Anita Elberse, and the authors would like to acknowledge her contribution and insights. They would also like to thank Barry Bayus, Stefan Stremersch, Marnik Dekimpe, and JB Steenkamp for helpful comments on earlier drafts. The usual disclaimer applies and all remaining errors are our own.

\section{References}

1. Bass FM (1969) A new product growth model for consumer durables. Manag Sci 15:215-227

2. Bass FM, Krishnan TV, Jain D (1994) Why the bass model fits without decision variables. Mark Sci 13(3):203-223

3. Basu A, Mazumdar T, Raj SP (2003) Indirect network externality effects on product attributes. Mark Sci 22(2):209-221

4. Bayus BL (1987) Forecasting sales of new contingent products: an application to the compact disc market. J Prod Innov Manag 4(4): 243-255

5. Bayus BL, Kim N, Shocker AD (2000) Growth models for multiproduct interactions: current status and new directions. In: Mahajan V, Muller E, Wind Y (eds) New-Product Diffusion Models. Kluwer, Boston, pp 141-164

6. Bucklin L, Sengupta S (1993) The co-diffusion of complementary innovations: supermarket scanners and UPC symbols. J Prod Innov Manag 10(March): 148-160

7. Dekimpe MG, Parker PM, Sarvary M (1998) Staged estimation of international diffusion models: an application to global cellular telephone adoption. Technol Forecast Soc Chang 57:105-132

8. Dekimpe MG, Parker PM, Sarvary M (2000) Global diffusion of technological innovations: a coupled-hazard approach. J Mark Res 37(1):47-59

9. Dekimpe MG, Parker PM, Sarvary M (2000) Multimarket and global diffusion in V. Mahajan. In: Muller E, Wind Y (eds) New-Product Diffusion. Kluwer, Boston

10. Economides N (1996) Economics of networks. Int J Ind Organ 14: 673-700

11. Fam KS, Merrilees B (1998) Cultural values and personal selling. Int Mark Rev 15(4):246-256

12. Gelder S, Stremersch S (2010) Variable selection in international new product growth. Manuscr

13. Goldenberg J, Libai B, Muller E (2009) The chilling effects of network externalities. Int J Res Mark 27(1):4-15

14. Goolsbee A, Klenow P (2002) Evidence on learning and network externalities in the diffusion of home computers. J Law Econ 45(2, part 1):317-344

15. Greene WH (1997) Econometric Analysis. Prentice Hall, Upper Saddle River
16. Gupta S, Jain D, Sawhney MS (1999) Modeling the evolution of markets with indirect network externalities: an application to the digital television. Mark Sci 18(3):396-416

17. Hofstede GH (1980) Culture's consequences: international differences in work-related values. Sage Publications, Beverly Hills

18. Hofstede GH (1991) Cultures and Organizations. McGraw-Hill, New York

19. Kale SH (1991) Culture-specific marketing communications: an analytical approach. Int Mark Rev 8(2):18-30

20. Katz M, Shapiro C (1994) Systems competition and network effects. J Econ Perspect 8(2):93-115

21. Lam D, Lee A, Mizerski R (2009) The effects of cultural values in word-of-mouth communication. J Int Mark 17(3):55-70

22. Liu BS, Furrer O, Sudharshan D (2001) The relationships between culture and behavioural intentions toward services. J Serv Res 4(2): $118-130$

23. Mahajan V, Muller E (1994) Innovation diffusion in a borderless global market: will the 1992 unification of the European community accelerate diffusion of new ideas, products and technologies? Technol Forecast Soc Chang 45:221-235

24. Mahajan V, Muller E, Wind Y (2000) New-Product Diffusion Models. Kluwer, Boston

25. Mortenson ST (2002) Sex, communication values, and cultural values: individualism-collectivism as a mediator of sex differences in communication values in two cultures. Commun Rep 15(1):57-71

26. Peterson R, Mahajan V (1978) Multi-product growth models. J Sheth (Editor), Research in Marketing (Vol. 1 pp. 201-231). JAI Press

27. Putsis WP, Balasubramanian S, Kaplan E, Sen SK (1997) Mixing behavior in cross-country diffusion. Mark Sci 16(4):354-369

28. Putsis WP, Srinivasan H (1994) Buying or just browsing? The duration of purchase Deliberation. J Mark Res 393-402

29. Rogers EM (1995) Diffusion of Innovations, 4th edn. The Free Press, New York

30. Rohlfs J (1974) A theory of interdependent demand for a communications service. Bell J Econ 5(1):16-37

31. Roset, Pere and Agusti Canals (2011), "A model of technology diffusion in separate and unrelated dual markets. Work Pap Draft

32. Shapiro C, Varian HR (1999) Information rules: a strategic guide to the network economy. Harv Bus Sch Press

33. Steenkamp JBEM, Hofstede FT, Wedel M (1999) A cross-national investigation into the individual and national cultural antecedents of consumer innovativeness. J Mark 63(2):55-69

34. Stremersch S, Binken JLG (2009) The effect of superstar software on hardware sales in system markets. J Mark Res

35. Stremersch S, Tellis GJ (2004) Understanding and managing international growth of new products. Int J Res Mark 21(4):421-439

36. Stremersch S, Tellis GJ, Franses PH, Binken JLG (2007) Indirect network effects in new product growth. J Mark 71(3):52-74

37. Sultan F, Farley JU, Lehmann DR (1990) A meta-analysis of applications of diffusion models. J Mark Res 27(1):70

38. Talukdar D, Sudhir K, Andrew A (2002) Investigating new product diffusion across products and countries. Mark Sci 21(1):97-114

39. Tellis GJ, Stremersch S, Yin E (2003) The international takeoff of new products: the role of economics, culture and country innovativeness. Mark Sci 22(2): 188-208

40. Tellis GJ, Yin Y, Niraj R (2009). Does quality win: network effects versus quality in high tech markets. J Mark Res

41. Van den Bulte C, Joshi YV (2007) New product diffusion with independents and imitators. Mark Sci 26(3):400-421

42. Van Everdingen Y, Stremersch S, Fok D (2009) Modeling global spill-over in new product takeoff. J Mark Res 46(5):637-652

43. Yeniyurt S, Townsend JD (2003) Does culture explain acceptance of new products in a country? An empirical investigation. Int Mark Rev 20(4):377-396 